\title{
Comparing Functional Paradigms for Exact Real-number Computation
}

\author{
Andrej Bauer ${ }^{1, \star}$, Martín Hötzel Escardó ${ }^{2}$, and Alex Simpson ${ }^{3, \star \star}$ \\ 1 IMFM, University of Ljubljana, Slovenia \\ 2 School of Computer Science, University of Birmingham, England \\ 3 LFCS, Division of Informatics, University of Edinburgh, Scotland
}

Version of January 21, 2002.

\begin{abstract}
We compare the definability of total functionals over the reals in two functional-programming approaches to exact real-number computation: the extensional approach, in which one has an abstract datatype of real numbers; and the intensional approach, in which one encodes real numbers using ordinary datatypes. We show that the type hierarchies coincide up to second-order types, and we relate this fact to an analogous comparison of type hierarchies over the external and internal real numbers in Dana Scott's category of equilogical spaces. We do not know whether similar coincidences hold at third-order types. However, we relate this question to a purely topological conjecture about the Kleene-Kreisel continuous functionals over the natural numbers. Finally, although it is known that, in the extensional approach, parallel primitives are necessary for programming total first-order functions, we demonstrate that, in the intensional approach, such primitives are not needed for second-order types and below.
\end{abstract}

\section{Introduction}

In functional programming, there are two main approaches to exact real-number computation. One is to use a specialist functional programming language that contains the real numbers as an abstract datatype. This approach is extensional in the sense that the data structures representating real numbers are hidden from view and one may only manipulate reals via representation-independent operations upon them. A second approach is to use an ordinary functional language, and to encode real numbers using standard infinite data structures, for example, streams. This approach is intensional in the sense that one has direct access to the encodings of reals, allowing the possibility of distinguishing between different representations of the same real number. In recent years, the extensional approach has been the subject of much theoretical investigation via the study of specialist languages, such as Di Gianantonio's RL [DiG93] and Escardó's RealPCF [Esc96]. On the other hand, the intensional approach is the

\footnotetext{
* Research supported by the Slovene Ministry of Science grant Z1-3138-0101-01

** Research supported by an EPSRC Advanced Research Fellowship.
} 
one that is actually used when exact real-number computation is implemented in practice - see, for example, [GL01].

This paper presents preliminary results in a general investigation relating the two approaches. Specifically, we address the question of how the programmability of higher-type total functionals over the real numbers compares between the two approaches. To this end, we consider two type hierarchies built using function space and product over a single base type, real. The first hierarchy is constructed by interpreting each type $\sigma$ as the set $[\sigma]_{E}$ of extensionally programmable total functionals of that type, and the second by interpreting $\sigma$ as the set $[\sigma]_{I}$ of intensionally programmable total functionals. As our first main result, Theorem 1, we prove that for all second-order (and below) types $\sigma$, the sets $[\sigma]_{E}$ and $[\sigma]_{I}$ coincide, thus a second-order functional is extensionally programmable if and only if it is intensionally programmable. This result thus applies at the type level at which definite integration,

$$
(f, a, b) \mapsto \int_{a}^{b} f(x) \mathrm{d} x \quad: \quad(\text { real } \rightarrow \text { real }) \times \text { real } \times \text { real } \rightarrow \text { real },
$$

resides; although, in the case of integration, our result gives no new information as it is already known to be programmable under both the extensional and intensional approaches, see [EE00] and [Sim98] respectively.

We prove Theorem 1 by relating it to an analogous question of the coincidence of type hierarchies in the setting of Dana Scott's category of equilogical spaces [Sco96,BBS02]. In that setting there is an external type hierarchy $(\sigma)_{E}$, built over Euclidean space, and there is an internal hierarchy $(\sigma)_{I}$, built over the object of real numbers as defined in the internal logic of the category. Again, we show that $(\sigma)_{E}$ and $(\sigma)_{I}$ coincide up to second-order types, Theorem 2 .

It is of course natural to ask whether the above type hierarchies also coincide for third-order $\sigma$ and above. We do not know the answer to this question, but a further contribution of this paper is to relate the agreement of the hierarchies at higher types to a purely topological conjecture about the Kleene-Kreisel continuous functionals [Kle59,Kre59] of second-order type, see Sect. 6.

Our methodology for studying the two approaches to exact real-number computation is to consider a paradigmatic programming language for each. For the extensional approach, we use Escardó's RealPCF+, which is RealPCF [Esc96] extended by a parallel existential operator - a language that enjoys the merit of being universal with respect to its domain-theoretic semantics [ES99]. For the intensional approach, we encode real numbers within Plotkin's PCF++, which is PCF extended by parallel-conditional and existential operators [Plo77]. Again, $\mathbf{P C F}++$ enjoys a universality property with respect to its denotational semantics [Plo77].

Admittedly, both RealPCF+ and $\mathbf{P C F}++$ are idealized languages, distant from real-world functional languages such as Haskell [Has]. As such, they provide ideal vehicles for a theoretical investigation into programmability questions such as ours. Nevertheless, it is our desire that our results should relate to the actual practice of exact real-number computation. There is one main obstacle to such 
a transference of the results: the parallel features of $\mathbf{P C F}++$ do not appear in Haskell and related languages. We address this issue in Sect. 9, where we show that, again for second-order $\sigma$ and below, the parallel features of PCF ++ are nowhere required to program functionals in $[\sigma]_{I}$, Theorem 3 . Thus a secondorder total functional over the reals is programmable in an ordinary sequential functional language if and only if it is programmable in the idealized, specialist and highly parallel language RealPCF+. Again, we do not know whether this result extends to third-order types and above.

Although our investigation is one into questions of programmability (i.e. of definability) within RealPCF + and $\mathbf{P C F}++$, we carry out the investigation purely at the denotational level, relying on known universality results to infer definability consequences from the semantic correspondences we establish. In doing so, there is one major way in which the results presented in this paper depart from the outline presented above. A full investigation would show that the computable (and hence definable) total functionals coincide between the denotational interpretations of the extensional and intensional approaches. Instead, we establish the coincidence of arbitrary total functionals, whether computable or not. Our reason for ignoring computability questions is that the results we establish already require significant technical machinery. Although it should be possible to give computability-sensitive versions of our results, this would unavoidably cause still further technical complications in the proofs. We leave this for future work. We remark that the results we prove, although computability free, do nonetheless have definability consequences relative to functional languages extended with oracles for all set-theoretic functions from $\mathbb{N}$ to $\mathbb{N}$, or equivalently relative to functional languages with programs given by infinite syntax trees.

The main question left open by this paper is whether our results extend to third-order types and beyond. Regarding this, we remark that we lack examples of genuinely interesting total functionals of type three to which such generalisations of our results would apply. However, another interesting development of our work would be to compare hierarchies of partial functionals over the reals.

This paper assumes some familiarity with domain theory, topology and category theory, for which our references are [AJ94,Dug89,Mac71] respectively. An important contribution of the paper is to show how mathematical tools from these subjects may be combined to attack seemingly innocuous questions that originate in functional programming.

\section{Domains for Real-number Computation}

We first fix terminology - see [AJ94] for definitions. We assume that a directedcomplete partial order (dcpo), $(D, \sqsubseteq)$, has least element. We call a dcpo $\omega$ continuous if it has a countable basis, and we write $x \ll y$ for the way-below relation on it. For us, a domain is an $\omega$-continuous bounded-complete dcpo. We write $\omega \mathbf{B C}$ for the category of domains and (directed-)continuous functions, and we write $\omega \mathbf{L}$ for its full subcategory of $\omega$-continuous lattices. Both categories are cartesian closed with exponentials given by the dcpo of all continuous functions. 
Our main interest will be in two particular domains, one for each of the two approaches to exact real-number computation mentioned in the introduction. The interval domain $\mathcal{I}$ has underlying set $\{\mathbb{R}\} \cup\{[a, b] \mid a \leq b \in \mathbb{R}\}$, with its order defined by $\delta \sqsubseteq \delta^{\prime}$ if and only if $\delta \supseteq \delta^{\prime}$. This is indeed a domain.

The interval domain is intimately connected with the extensional approach to exact real-number computation. Indeed, the abstract datatype of real numbers in RealPCF [Esc96] is specifically designed to have $\mathcal{I}$ as its denotational interpretation. Furthermore, Escardó and Streicher [ES99] have established a universality result with respect to the domain-theoretic semantics: every computable element in the domain interpreting a RealPCF type is definable, by a term of that type, in the language RealPCF +, which is RealPCF extended with a parallel existential operator. In this paper, although we are motivated by definability questions, we do not wish to entangle ourselves in computability issues. Thus we remark on the following modified version of Escardó and Streicher's result. Every element (computable or not) in the domain interpreting a RealPCF type is definable in the language $\Omega$ RealPCF+, which is RealPCF+ extended with an oracle for every set-theoretic function from $\mathbb{N}$ to $\mathbb{N}$.

Under the intensional approach to exact real-number computation, one needs to select a computationally admissible representation of real numbers [WK87]. There are many equivalent choices. For simplicity, we use a mantissa-exponent representation, where the mantissa, a real number in the interval $[-1,1]$, is represented using signed-binary expansions. Specifically, a real number is represented by a pair $(n, \alpha)$ where the mantissa $\alpha \in\{-1,0,1\}^{\omega}$ represents the number $0 . \alpha_{0} \alpha_{1} \alpha_{2} \ldots$, i.e. $\sum_{i=0}^{\infty} 2^{-(i+1)} \alpha_{i}$, and the exponent $n \in \mathbb{N}$ gives a multiplier of $2^{n}$, thus the pair $(n, \alpha)$ represents the real number $\sum_{i=0}^{\infty} 2^{n-(i+1)} \alpha_{i}$.

To implement the above representation in a functional programming language, one would most conveniently encode a real number as a pair consisting of a natural number followed by a stream. However, in order to fix on as simple a language as possible, we use instead a direct implementation in Plotkin's PCF [Plo77] extended with product types. In PCF, the base type, nat, is interpreted as the flat domain $\mathbb{N}_{\perp}=\{\perp\} \cup \mathbb{N}$ with least element $\perp$. Function space and product are interpreted using the cartesian-closed structure of $\omega \mathbf{B C}$. As we are interested in definability, we mention Plotkin's universality result: every computable element in the domain interpreting a PCF type is definable in the language $\mathbf{P C F}++$, which is $\mathbf{P C F}$ extended with parallel-conditional and existential operators. Again, there is a computability-free version of this result. Every element (computable or not) in the domain interpreting a PCF type is definable in the language $\Omega \mathbf{P C F}++$, which is $\mathbf{P C F}++$ extended with an oracle for every set-theoretic function from $\mathbb{N}$ to $\mathbb{N}$.

We represent real numbers, in $\mathbf{P C F}$, using the type nat $\rightarrow$ nat whose denotational interpretation is the function domain $\mathcal{J}=\mathbb{N}_{\perp} \mathbb{N}_{\perp}$. We say that a function $f \in \mathcal{J}$ is real representing if $f(0) \neq \perp$ and if $f(x) \in\{0,1,2\}$ when $x>0$. Any such real-representing $f$ encodes the real number $\sum_{i=1}^{\infty} 2^{f(0)-i}(f(i)-1)$. 


\section{Two Type Hierarchies of Assemblies}

Our goal is to investigate the type hierarchies of total functionals on reals programmable in the two approaches to exact real-number computation. We consider simple types over a base type of real numbers, with types given by:

$$
\sigma::=\text { real }\left|\sigma \times \sigma^{\prime}\right| \sigma \rightarrow \sigma^{\prime} .
$$

The order of a type is: $\operatorname{order}($ real $)=0$; $\operatorname{order}\left(\sigma \times \sigma^{\prime}\right)=\max \left(\operatorname{order}(\sigma), \operatorname{order}\left(\sigma^{\prime}\right)\right)$; and $\operatorname{order}\left(\sigma \rightarrow \sigma^{\prime}\right)=\max \left(1+\operatorname{order}(\sigma), \operatorname{order}\left(\sigma^{\prime}\right)\right)$.

For the extensional approach, we study the total functionals on reals programmable in the language $\Omega$ RealPCF + . Because of the universality result mentioned above, such functionals are exactly those total functionals that arise in the type hierarchy over $\mathcal{I}$ in $\omega \mathbf{B C}$. However, the type hierarchy over $\mathcal{I}$ contains both superfluous elements and redundancies. For example, $\mathcal{I}$ itself contains "partial" real numbers (proper intervals) in addition to "total" reals (singleton intervals). At first-order types, such as $\mathcal{I}^{\mathcal{I}}$, there are elements that do not represent total functions on reals because they fail to preserve total reals. Furthermore, at the same type, it is possible to have two different functions $f, g: \mathcal{I} \rightarrow \mathcal{I}$ that represent the same total function on reals, because, although they behave identically on total reals, they differ in their behaviour on partial reals.

For the intensional approach, we study the functionals programmable in $\Omega \mathbf{P C F}++$, using the representation described in Sect. 2. Because of the universality result, such functionals are exactly those total functionals that arise in the type hierarchy over $\mathcal{J}$ in $\omega \mathbf{B C}$. Again, there is superfluity and redundancy. Within $\mathcal{J}$, we singled out the real-representing elements in Sect. 2, and in fact each real number has infinitely many different representations. Because of this, there are two ways that a function from $\mathcal{J}$ to $\mathcal{J}$ may fail to represent a function on real numbers: either it may map some real-representing element to a non-real-representing element; or it may map two different representations of the same real number to representations of different real numbers.

Assemblies offer a convenient way of identifying the elements of the hierarchies over $\mathcal{I}$ and $\mathcal{J}$ in $\omega \mathbf{B C}$ that represent total functionals on reals. An assembly is a triple $A=\left(|A|,\|A\|, \vdash_{A}\right)$ where $|A|$ is a set, $\|A\|$ is a domain, and $\vdash_{A}$ is a binary relation between $\|A\|$ and $|A|$ such that, for all $a \in|A|$, there exists $x \in\|A\|$ such that $x \Vdash_{A} a$. A morphism from one assembly $A$ to another $B$ is simply a function $f:|A| \rightarrow|B|$ for which there exists a continuous $g:\|A\| \rightarrow\|B\|$ such that $x \Vdash_{A} a$ implies $g(x) \Vdash_{B} f(a)$, in which case we say that $g$ tracks $f$. We write $\operatorname{Asm}(\omega \mathbf{B C})$ for the category of assemblies over domains, and $\mathbf{A s m}(\omega \mathbf{L})$ for the full subcategory of assemblies over $\omega$-continuous lattices. Again, both categories are cartesian closed, with the exponential $B^{A}$ given by

$$
\begin{aligned}
\left|B^{A}\right| & =\{f:|A| \rightarrow|B| \mid f \text { is a morphism from } A \text { to } B\} \\
\left\|B^{A}\right\| & =\|B\|^{\|A\|} \text { in } \omega \mathbf{B C} \\
g \Vdash_{B^{A}} f & \Longleftrightarrow g \text { tracks } f .
\end{aligned}
$$


We remark that $\mathbf{A s m}(\omega \mathbf{B C})$ is equivalent to the category $\mathbf{A s m}(\mathrm{U})$ of assemblies over the combinatory algebra given by $\mathrm{U}$, a chosen universal domain [GS90]; and $\operatorname{Asm}(\omega \mathbf{L})$ is equivalent to $\mathbf{A} \mathbf{s m}(P)$, for Scott's combinatory algebra $P=\mathcal{P} \omega$ which is an $\omega$-continuous lattice under $\subseteq$. Thus, up to equivalence, $\operatorname{Asm}(\omega \mathbf{B C})$ and $\operatorname{Asm}(\omega \mathbf{L})$ arise as the categories of double-negation separated objects in the realizability toposes $\mathbf{R T}(\mathrm{U})$ and $\mathbf{R T}(\mathrm{P})$ respectively.

We use $\mathbf{A s m}(\omega \mathbf{B C})$ to define the two type hierarchies of total functionals we are interested in. For the extensional approach, we define an assembly $\llbracket \sigma \rrbracket_{E}$ for each type $\sigma$. For the base type, real, this is given by:

$$
\mid \llbracket \text { real } \rrbracket_{E} \mid=\mathbb{R} \quad \| \llbracket \text { real } \rrbracket_{E} \|=\mathcal{I} \quad \delta \Vdash_{\llbracket \text { real } \rrbracket_{E}} x \Longleftrightarrow \delta=\{x\}
$$

from which $\llbracket \sigma \rrbracket_{E}$ is defined for arbitrary $\sigma$ using the cartesian-closed structure of $\operatorname{Asm}(\omega \mathbf{B C})$.

For each type $\sigma$, the set $\left|\llbracket \sigma \rrbracket_{E}\right|$, for which we henceforth use the less cluttered $[\sigma]_{E}$, is a set of total functionals of appropriate type over the reals. By the universality of $\Omega$ RealPCF+, the functionals $f \in[\sigma]_{E}$ are exactly those for which there exists an $\Omega$ RealPCF + program $P$ of type $\sigma$ such that $P$ computes $f$, as witnessed by the relation $\llbracket P \rrbracket \Vdash_{\llbracket \sigma} \rrbracket_{E} f$, where $\llbracket P \rrbracket$ is the denotational interpretation of $P$.

Similarly, for the intensional approach, the assembly $\llbracket$ real $\rrbracket_{I}$ is defined by:

$$
\mid \llbracket \text { real } \rrbracket_{I} \mid=\mathbb{R} \quad \| \llbracket \text { real } \rrbracket_{I} \|=\mathcal{J} \quad f \Vdash_{\llbracket \text { real } \rrbracket_{I} x \Longleftrightarrow} \begin{aligned}
& f \text { is real representing and } \\
& x=\sum_{i=1}^{\infty} 2^{f(0)-i} \cdot(f(i)-1)
\end{aligned}
$$

and again $\llbracket \sigma \rrbracket_{I}$ is induced for arbitrary $\sigma$ using the cartesian-closed structure of $\operatorname{Asm}(\omega \mathbf{B C})$. This time the set $\left|\llbracket \sigma \rrbracket_{I}\right|$, for which we henceforth write $[\sigma]_{I}$, is the set of those total functionals $f$ for which there exists an $\Omega \mathbf{P C F}++$ program $P$ of type $\sigma^{*}$ (where real ${ }^{*}=$ nat $\rightarrow$ nat and $(\cdot)^{*}$ commutes with function space and product) such that $P$ computes $f$, as witnessed by the relation $\llbracket P \rrbracket \Vdash_{\llbracket \sigma \rrbracket_{I}} f$.

We can now state our first result, the agreement of the two hierarchies for second-order types and below.

Theorem 1. For any type $\sigma$ with $\operatorname{order}(\sigma) \leq 2$, it holds that $[\sigma]_{E}=[\sigma]_{I}$.

Although this theorem can be proved directly, we find it illuminating to approach it by looking at another situation in which there are competing hierarchies of total functionals over the reals. This will occupy Sects. 4-6, after which the proof of Theorem 1 will be given in Sect. 7 .

\section{Two Type Hierarchies of Equilogical Spaces}

Our second example of a pair of hierarchies of real functionals arises in Dana Scott's category of equilogical spaces [Sco96,BBS02], a cartesian-closed extension of the category of topological spaces. In addition to its application to Theorem 1, our study of the two type hierarchies in this setting is of independent interest. 
In the present paper we only consider countably-based equilogical spaces, and we do not impose Scott's $T_{0}$ requirement. For our purposes then, an equilogical space is a triple $X=\left(|X|,\|X\|, q_{X}\right)$ where $|X|$ is a set, $\|X\|$ is a countably-based (i.e. second-countable) topological space and $q_{X}:\|X\| \rightarrow|X|$ is a surjective function. A morphism from one equilogical space $X$ to another $Y$ is simply a function $f:|X| \rightarrow|Y|$ for which there exists a continuous $g:\|X\| \rightarrow\|Y\|$ such that $q_{Y} \circ g=f \circ q_{X}$. Again we say that $g$ tracks $f$. We write $\omega \mathbf{E q u}$ for the category of equilogical spaces.

We write $\omega$ Top for the category of countably-based topological spaces. There is a full and faithful functor from $\omega$ Top to $\omega \mathbf{E q u}$, mapping a countably-based space $S$ to $\left(S, S\right.$, id $\left._{S}\right)$.

There are two non-isomorphic equilogical spaces, each with good claims to be the equilogical space of real numbers. The external reals, $\mathrm{R}_{\mathrm{E}}$, is the inclusion of the topological Euclidean reals as the object $\left(\mathbb{R}, \mathbb{R}, \mathrm{id}_{\mathbb{R}}\right)$. The internal reals, $\mathrm{R}_{\mathrm{l}}$, is the object $\left(\mathbb{N} \times 3^{\omega}, \mathbb{R}, r\right)$, where $3=\{-1,0,1\}$ with the discrete topology, both $3^{\omega}$ and $\mathbb{N} \times 3^{\omega}$ are given the product topologies, and $r$ is the function:

$$
\mathrm{r}(n, \alpha)=\sum_{i=0}^{\infty} 2^{n-(i+1)} \alpha_{i} .
$$

Thus, the internal reals are again based on the intensional signed-digit notation.

A remarkable fact is that is $\omega \mathbf{E q u}$ is equivalent to the category $\operatorname{Asm}(\omega \mathbf{L})$ [BBS02].

$$
\omega \mathbf{E q u} \underset{F}{\stackrel{P}{\rightleftarrows}} \operatorname{Asm}(\omega \mathbf{L})
$$

Here $P(X)$ is obtained from a topological pre-embedding $e:\|X\| \rightarrow \mathrm{P}$, where $\mathrm{P}=\mathcal{P} \omega$ is endowed with the Scott topology, by defining $\|P(X)\|=\mathrm{P},|P(X)|=$ $|X|$, and $x \Vdash_{P(X)} a$ if and only if there exists $z \in\|X\|$ with $e(z)=x$ and $q_{X}(z)=a$. (Recall that a continuous $e: S \rightarrow T$ between topological spaces is a pre-embedding if the open sets of $S$ are exactly $\left\{e^{-1}(U) \mid U \subseteq T\right.$ is open $\}$.) Conversely, for an assembly $A$ in $\operatorname{Asm}(\omega \mathbf{L}), F(A)$ is defined by $\|F(A)\|=$ $\sum_{a \in|A|}\{x \in\|A\| \mid x \Vdash a\}$ with the final topology as induced, from the Scott topology on $\|A\|$, by the second projection $\|F(A)\| \rightarrow\|A\|$. (Recall that the final topology is the unique topology with respect to which the function is a topological pre-embedding.) Then $|F(A)|=|A|$ and $q_{F(A)}$ is the first projection $\|F(A)\| \rightarrow|A|$.

The next proposition, which says that $R_{\mathrm{I}}$ is the object of reals as described in the internal logic of the topos $\mathbf{R T}(\mathrm{P})$, explains our nomenclature for $\mathrm{R}_{\mathbf{l}}$.

Proposition 1. The object $\mathrm{R}_{\mathrm{l}}$, when transported along

$$
\omega \mathbf{E q u} \stackrel{\simeq}{\longrightarrow} \operatorname{Asm}(\omega \mathbf{L}) \stackrel{\simeq}{\longrightarrow} \operatorname{Asm}(\mathrm{P}) \longrightarrow \mathbf{R T}(\mathrm{P})
$$

gives the object of Dedekind (equivalently Cauchy) reals in $\mathbf{R T}(\mathrm{P})$.

An important consequence of the equivalence between $\omega \mathbf{E q u}$ and $\mathbf{A s m}(\omega \mathbf{L})$ is that $\omega \mathbf{E q u}$ is cartesian closed. We use the cartesian-closed structure to determine two type hierarchies, the external $\left([\sigma)_{E}\right.$, and the internal $\left([\sigma)_{I}\right.$ in $\omega \mathbf{E q u}$, 
defined at base type by:

$$
(\text { real })_{E}=\mathrm{R}_{\mathrm{E}} \quad\left([\text { real })_{I}=\mathrm{R}_{\mathrm{I}} .\right.
$$

We write $(\sigma)_{E}$ as an abbreviation for $\left|([\sigma])_{E}\right|$, and $(\sigma)_{I}$ for $\left|([\sigma])_{I}\right|$.

Theorem 2. For any type $\sigma$ with $\operatorname{order}(\sigma) \leq 2$, it holds that $(\sigma)_{E}=(\sigma)_{I}$.

\section{Topological Considerations}

We shall need to consider various types of topological spaces. Recall that a topological space is said to be regular if every neighbourhood $W$ of a point $x$ has a closed subneighbourhood, i.e., there exist $U \subseteq V \subseteq W$ such $V$ is closed, $U$ is open and $x \in U$. A space is said to be zero-dimensional if every neighbourhood of a point has a clopen subneighbourhood, where a clopen set is one that is both open and closed. Clearly every zero-dimensional space is regular. Some useful countably-based zero-dimensional spaces are: Cantor space, $2^{\omega}$ with the product topology; Baire space, $\mathbb{N}^{\omega}$ with the product topology; and the one-point compactification of $\mathbb{N}$, the set $\mathbb{N}^{+}=\mathbb{N} \cup\{\infty\}$ with topology given by the clopen basis $\{\{n\} \mid n \in \mathbb{N}\} \cup\{\{m \mid m \geq n\} \cup\{\infty\} \mid n \in \mathbb{N}\}$. We shall need the following result about zero-dimensional spaces.

Proposition 2. If $S$ is a nonempty closed subspace of a countably-based zerodimensional space $T$ then $S$ is a retract of $T$.

Proof. Because $T$ is countably-based and zero-dimensional it is pre-embedded into Cantor space by an embedding $e: T \rightarrow 2^{\omega}$. Such an embedding induces a countable clopen basis on $T$, defined by $U_{a}=e^{-1}([a])$ where $a \in 2^{*}$ is a finite binary sequence and $[a]$ is the set of all infinite binary sequences starting with $a$. For each $a \in 2^{*}$ such that $U_{a} \cap S \neq \emptyset$, pick a point $c_{a} \in U_{a} \cap S$. A continuous retraction $r: T \rightarrow S$ is defined as follows. For $x \in T$, consider the set $A_{x}=\left\{a \in 2^{*} \mid x \in U_{a} \wedge U_{a} \cap S \neq \emptyset\right\}$. The set $A_{x}$ is nonempty because $S$ is nonempty, it is prefix-closed, and if $a, b \in A_{x}$ then $a$ is a prefix of $b$ or $b$ is a prefix of $a$. If $A_{x}$ is infinite, then every basic open neighbourhood of $x$ intersects the closed set $S$, which means that $x \in S$ and so we define $r(x)=x$. Otherwise, there is a longest $a \in A_{x}$ and every other element of $A_{x}$ is a prefix of $a$. In this case we define $r(x)=c_{a}$. Clearly, $r$ is a retraction, and it remains to verify that it is continuous. For that purpose, we show that the inverse image $r^{-1}\left(U_{a}\right)$ of a basic open is open. Suppose $x \in r^{-1}\left(U_{a}\right)$. If $x \notin S$ then there exists $U_{b} \ni x$ such that $U_{b} \cap S=\emptyset$ and we have $x \in U_{b} \subseteq r^{-1}\left(U_{a}\right)$ because $r(y)=r(x)$ for all $y \in U_{b}$. The other possibility is that $x \in S$, but then $r(y) \in U_{a}$ for all $y \in U_{a}$, therefore $x \in U_{a} \subseteq r^{-1}\left(U_{a}\right)$.

In a topological space $T$, an infinite sequence $\left(x_{i}\right)_{i \geq 0}$ converges to a point $x$, notation $\left(x_{i}\right) \rightarrow x$, if, for all neighbourhoods $U \ni x$, the sequence $\left(x_{i}\right)$ is eventually in $U$ (i.e., there exists $l \geq 0$ such that $x_{j} \in U$ for all $j \geq l$ ). A 
subset $X \subseteq T$ is sequentially open if, whenever $\left(x_{i}\right) \rightarrow x \in X$, it holds that $\left(x_{i}\right)$ is eventually in $X$. Every open set is sequentially open. A space $T$ is said to be sequential if every sequentially open subset is open. We write Seq for the category of sequential spaces. This category is known to be cartesian closed. If $S$ and $T$ are sequential then the exponential $T^{S}$ is given by the set of all continuous functions endowed with the unique sequential topology that induces the convergence relation $\left(f_{i}\right) \rightarrow f$ if and only if, whenever $\left(x_{i}\right) \rightarrow x$ in $S$, it holds that $\left(f_{i}\left(x_{i}\right)\right) \rightarrow f(x)$ in $T$. We shall need the following useful facts, where $2=\{0,1\}$ with the discrete topology, $X^{\mathbb{N}}$ is the exponential of $X$ and $\mathbb{N}$ in Seq, whereas $X^{\omega}$ is a countable product of copies of $X$ with the product topology, as above.

Proposition 3. In the category Seq,

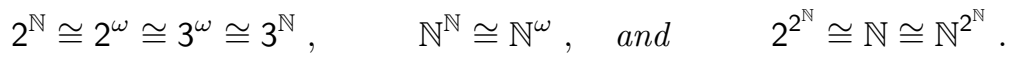

Proof. Because all spaces are of the form $X^{Y}$ where $X$ is discrete and $Y$ is both compact and locally compact.

We write $\omega$ qTop for the category of all quotient spaces of countably-based spaces, i.e. a topological space $T$ is an object of $\omega \mathbf{q T o p}$ if and only if there exists a countably-based space $S$ with a topological quotient $q: S \rightarrow T$. There are subcategory inclusions $\omega$ Top $\hookrightarrow \omega \mathbf{q T o p} \hookrightarrow$ Seq. Importantly, the category $\omega \mathbf{q T o p}$ is cartesian closed with its cartesian-closed structure inherited from Seq [MS02].

A topological space is said to be hereditarily Lindelöf if, for every family $\left\{U_{i}\right\}_{i \in I}$ of open sets, there is a countable subfamily $\left\{U_{j}\right\}_{j \in J}$ (i.e. where $J \subseteq I$ is countable) such that $\bigcup_{j \in J} U_{j}=\bigcup_{i \in I} U_{i}$. It is easily shown that every space in $\omega \mathbf{q T o p}$ is hereditarily Lindelöf.

The next proposition relates the above notions to an important property of the function $r: \mathbb{N} \times 3^{\omega} \rightarrow \mathbb{R}$, defined in (1), which is a topological quotient. We first introduce terminology that makes sense in an arbitrary category. Given an object $Z$ and a morphism $g: X \longrightarrow Y$ we say that $Z$ is $g$-projective, or equivalently that $g$ projects $Z$, if, for every $f: Z \longrightarrow Y$, there exists $\bar{f}: Z \rightarrow X$ such that the left-hand diagram below commutes. Dually, we say that $Z$ is $g$ injective, or equivalently that $g$ injects $Z$, if, for every $f: X \longrightarrow Z$, there exists $\bar{f}: Y \rightarrow Z$ such that the right-hand diagram commutes.
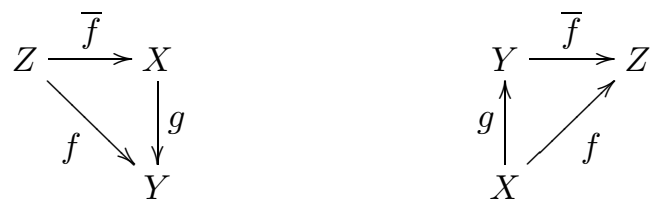

A useful fact is that if $Y$ is a sequential topological space and $g$ projects $\mathbb{N}^{+}$ then $g$ is a topological quotient map.

Proposition 4. If $T$ is a hereditarily Lindelöf space then $T$ is zero-dimensional if and only if it is both regular and r-projective. 
Proof. In the proof we rely on the fact that a Hereditarily Lindelöf space is regular if and only if it is completely normal, cf. [Dug89, Exercise VIII.6.7, p. 180], and thus also completely regular.

Suppose $T$ is hereditarily Lindelöf, regular, and r-projective. Let $x \in U$ be a point with an open neighbourhood. Because $T$ is also completely regular, there exists $f: T \rightarrow \mathbb{R}$ such that $f(x)=0$ and $V=f^{-1}((-1,1))$ is contained in $U$. Because $T$ is r-projective, there exists $\bar{f}: T \rightarrow \mathbb{N} \times 3^{\omega}$ such that $f=\mathrm{r} \circ \bar{f}$. Then $W=\bar{f}^{-1}\left(\{0\} \times\left\{a \in 3^{\omega} \mid a(0)=1\right\}\right)$ is a clopen neighbourhood of $x$ such that $W \subseteq V \subseteq U$. Therefore, $T$ is zero-dimensional.

Conversely, suppose $T$ is hereditarily Lindelöf and zero-dimensional. Then it is regular, and we need to show that it is r-projective. First we note that every countable open cover $\left\{U_{i} \mid i \in \mathbb{N}\right\}$ of $T$ has a precise disjoint refinment, i.e., a clopen cover $\left\{V_{i} \mid i \in \mathbb{N}\right\}$ such that $V_{i} \subseteq U_{i}$ for all $i \in \mathbb{N}$, and $V_{i} \cap V_{j}=\emptyset$ when $i \neq j$. Now suppose $f: T \rightarrow \mathbb{R}$ is a continuous map. We construct a lifting $\bar{f}: T \rightarrow \mathbb{N} \times 3^{\omega}$ by defining each of its components $\bar{f}_{1}: T \rightarrow \mathbb{N}$ and $\bar{f}_{2}: T \rightarrow 3^{\omega}$ separately. Consider the open cover of $T$

$$
U_{i}=f^{-1}\left(\operatorname{int}\left(r\left(\{i\} \times 3^{\omega}\right)\right)\right) \quad \text { where } i \in \mathbb{N} .
$$

There exists a precise disjoint refinment $\left\{W_{i} \mid i \in \mathbb{N}\right\}$. Define $\bar{f}_{1}(x)$ to be the unique $i \in \mathbb{N}$ for which $x \in W_{i}$. The second component $\bar{f}_{2}$ is defined separately on each $W_{i}$, as follows. For $j \in \mathbb{N}$, consider the open cover $\left\{V_{j, 0}, V_{j, 1}, V_{j, 2}\right\}$ of $W_{i}$, defined by

$$
V_{j, d}=f^{-1}\left(\operatorname{int}\left(r\left(\{i\} \times\left\{a \in 3^{\omega} \mid a(j)=d\right\}\right)\right)\right) .
$$

There is a precise disjoint refinment $\left\{W_{j, 0}^{\prime}, W_{j, 1}^{\prime}, W_{j, 2}^{\prime}\right\}$. For $x \in W_{i}$, define $\bar{f}_{2}(x)(j)$ to be the unique $d \in\{0,1,2\}$ for which $x \in W_{j, d}^{\prime}$. The map $\bar{f}(x)=$ $\left(\bar{f}_{1}(x), \bar{f}_{2}(x)\right)$ is continuous because $\bar{f}_{1}$ and $\bar{f}_{2}$ are. It remains to be shown that $f=\mathrm{r} \circ \bar{f}$. Let $x \in T, i=\bar{f}_{1}(x)$, and $b=\bar{f}_{2}(x)$. By construction, for every $j \in \mathbb{N}, f(x) \in \mathrm{r}\left(\{i\} \times\left\{a \in 3^{\omega} \mid a(j)=b(j)\right\}\right)$, hence $f(x)=\mathrm{r}(i, b)=\mathrm{r}(\bar{f}(x))$, as required.

We end this section by relating $\omega \mathbf{q T o p}$ to $\omega \mathbf{E q u}$. Interestingly, $\omega \mathbf{q T o p}$ is equivalent to a full reflective subcategory of $\omega \mathbf{E q u}$. The faithful reflection functor $Q: \omega \mathbf{E q u} \rightarrow \omega \mathbf{q T o p}$ is easily described. It maps an equilogical space $X$ to the topological space $|X|$ obtained by endowing it with the quotient topology under the surjection $q_{X}:\|X\| \rightarrow|X|$. Its right adjoint, the full and faithful functor from $\omega \mathbf{q T o p}$ to $\omega \mathbf{E q u}$ requires further definitions. We say that a continuous funtion $q: S \rightarrow T$ between topological spaces is $\omega$-projecting if projects every countably-based space. The following proposition is due to M. Schröder [MS02].

Proposition 5. For every $T$ in $\omega \mathbf{q T o p}$ there exists a countably-based space $S_{T}$ and an $\omega$-projecting "cover" $q_{T}: S_{T} \rightarrow T$.

The functor $C: \omega \mathbf{q T o p} \rightarrow \omega \mathbf{E q u}$ maps $T$ to the equilogical space $\left(S_{T}, T, q_{T}\right)$. This functor is full and faithful and, moreover, preserves the cartesian-closed structure [MS02]. 
It will be helpful to have an improved understanding of how arbitrary exponentials in $\omega$ Equ relate to those in $\omega \mathbf{q T o p}$. Suppose then that $X$ and $Y$ are equilogical spaces. Let $q: W \rightarrow\|Y\|^{\|X\|}$ be an $\omega$-projecting cover of the $\omega \mathbf{q T o p}$ exponential $\|Y\|^{\|X\|}$ by a countably-based space $W$. Let $F \subseteq\|Y\|^{\|X\|}$ be the subset of functions that track morphisms from $X$ to $Y$. Then $F$ is a sequential space, with its topology determined by inheriting sequence convergence from $\|Y\|^{\|X\|}$ (the subsequential toplogy - in general this is finer than the subspace topology). Obtain $q^{\prime}: R \rightarrow F$ by the pullback in $\omega \mathbf{q T o p}$ below.

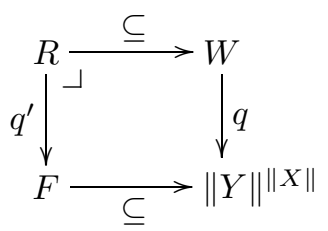

Then $R$ is a subspace of $W$, because the subsequential and subspace topologies agree on subsets of a countably-based space. Also, $q^{\prime}$ is $\omega$-projecting, as such maps are stable under pullback. Define $q^{\prime \prime}: F \rightarrow\left|Y^{X}\right|$ to be the function that maps $f \in F$ to the unique morphism tracked by it.

Proposition 6. Given equilogical spaces $X$ and $Y$, the exponential $Y^{X}$ in $\omega \mathbf{E q u}$ is isomorphic to $\left(\left|Y^{X}\right|, R, q^{\prime \prime} \circ q^{\prime}\right)$ as defined above.

Proof. First of all, the set $\left|Y^{X}\right|$ of all morphisms $Y \rightarrow X$ really is the right underlying set for the exponential $Y^{X}$ because $\omega \mathbf{E q u}$ is well-pointed and the forgetful functor $\omega \mathbf{E q u} \rightarrow$ Set maps $X$ to $|X|$. Given a function $f:|Z| \times|X| \rightarrow$ $|Y|$ that is tracked by some $g:\|Z\| \times\|X\| \rightarrow\|Y\|$, we need to show that there is a unique tracked function $\widetilde{f}:|Z| \rightarrow\left|Y^{X}\right|$ such that $\widetilde{f}(z)(x)=f(z, x)$ for all $z \in|Z|$ and $x \in|X|$. The existence and uniqueness of such a function are straightforward so we show that $\widetilde{f}$ is tracked. Consider the transpose $\widetilde{g}:\|Z\| \rightarrow\|Y\|^{\|X\|}$ in $\omega \mathbf{q T o p}$. Because $W \rightarrow\|Y\|^{\|X\|}$ is an $\omega$-projecting cover, $\widetilde{g}$ lifts through it as $h:\|Z\| \rightarrow W$. A simple diagram chase establishes that $h$ maps into $R$ and that it tracks $\tilde{f}$.

Now consider the equivalence relation $\sim_{Y}$ on $\|Y\|$ defined by

$$
x \sim_{Y} y \Longleftrightarrow q_{Y}(x)=q_{Y}(y) .
$$

Proposition 7. Given equilogical spaces $X$ and $Y$ such that $\sim_{Y} \subseteq\|Y\| \times\|Y\|$ is closed w.r.t. the product topology, the subset $F \subseteq\|Y\|^{\|X\|}$ is closed, hence the subspace topology on $F$ coincides with the subsequential topology.

Proof. The subset $F$ is equal to an intersection of closed sets:

$$
F=\bigcap_{a \sim_{X} b}\left\{f \in\|Y\|^{\|X\|} \mid f(a) \sim_{Y} f(b)\right\} .
$$




\section{Proof of Theorem 2}

We write $\omega \mathbf{0 E q u}$ for the full subcategory of $\omega \mathbf{E q u}$ consisting of those equilogical spaces that are isomorphic to one $X$ for which $\|X\|$ is zero-dimensional. Easily, $\omega \mathbf{0 E q u}$ is closed under finite products, and contains every countably-based zerodimensional space under the inclusion of $\omega$ Top in $\omega \mathbf{E q u}$, including $\mathbb{N}^{+}$. We say that a morphism $e: X \rightarrow Y$ in $\omega \mathbf{E q u}$ is tight if it is mono and it projects every space in $\omega \mathbf{0 E q u}$.

\section{Lemma 1.}

1. Every tight morphism is epi (as well as mono).

2. Every isomorphism is tight.

3. If $e: X \rightarrow Y$ and $f: Y \rightarrow Z$ are tight then $f \circ e$ is tight.

4. If $e: Y \rightarrow Z$ is tight then the pullback $f^{*}(e): X \times_{Z} Y \rightarrow X$ is tight for any $f: X \rightarrow Z$.

5. If $e: X \rightarrow Y$ is tight and $Z$ is in $\omega \mathbf{0 E q u}$ then $e^{Z}: X^{Z} \rightarrow Y^{Z}$ is tight.

6. If $e: X \rightarrow Y$ is tight then $e:|X| \rightarrow|Y|$ is a bijection and $Q e: Q X \rightarrow Q Y$ (using the functor $Q: \omega \mathbf{E q u} \rightarrow \omega \mathbf{q T o p}$ ) is a homeomorphism.

7. The "identity" $\mathrm{R}_{\mathrm{I}} \rightarrow \mathrm{R}_{\mathrm{E}}$ (tracked by $\mathrm{r}$ ) is tight.

Proof. We briefly remark on the reasons for the less obvious claims.

5. Because for any $W$ in $\omega \mathbf{0 E q u}, W \times Z$ is in $\omega \mathbf{0 E q u}$.

6. The homeomorphism is because $\mathbb{N}^{+}$is in $\omega \mathbf{0 E q u}$.

7. By Proposition 4.

We say that an equilogical space is tight-injective if it is injective with respect to every tight map. (N.b. as every tight morphism is epi, the extension demanded in the definition of injectivity is necessarily unique.) We write $\omega \mathbf{E} \mathbf{q} \mathbf{u}_{\mathbf{t i}}$ for the full subcategory of tight-injective objects in $\omega \mathbf{E q u}$.

\section{Lemma 2.}

1. $\omega \mathbf{E q u}_{\mathbf{t i}}$ is cartesian closed with its structure inherited from $\omega \mathbf{E q u}$.

2. Every space in $\omega \mathbf{T o p}$ is contained in $\omega \mathbf{E q u}_{\mathbf{t i}}$ under the inclusion functor $\omega \mathbf{T o p} \hookrightarrow \omega \mathbf{E q u}$.

Proof. 1. Because if $e: X \rightarrow Y$ is tight then so is $\left(\operatorname{id}_{Z} \times e\right): Z \times C \rightarrow Z \times Y$ by Lemma 1.4 .

2. Given a tight $e: X \rightarrow Y$ and a morphism $f: X \rightarrow T$ where $T$ is in $\omega$ Top, define $\bar{f}:|Y| \rightarrow T$ to be the unique function such that $\bar{f} \circ e=f$. We show that the function $\bar{f} \circ q_{Y}:\|Y\| \rightarrow T$ preserves convergent sequences, and is thus continuous, and hence tracks $\bar{f}$. Indeed, if $\left(x_{i}\right) \rightarrow x_{\infty}$ in $\|Y\|$ then there is a function $x_{(-)}: \mathbb{N}^{+} \rightarrow\|Y\|$, hence a morphism $h: \mathbb{N}^{+} \rightarrow Y$ tracked by $x_{(-)}$. Because $e$ is tight, there exists $\bar{h}: \mathbb{N}^{+} \rightarrow X$ such that $e \circ \bar{h}=h$. But then $f \circ \bar{h}: \mathbb{N}^{+} \rightarrow T$, which maps $l \in \mathbb{N} \cup\{\infty\}$ to $\bar{f}\left(q_{Y}\left(x_{l}\right)\right)$, shows that indeed $\bar{f}\left(q_{Y}\left(x_{i}\right)\right) \rightarrow \bar{f}\left(q_{Y}\left(x_{\infty}\right)\right)$. 
By the above lemma, every object $\left([\sigma)_{E}\right.$ is tight-injective, because $R_{E}$ is in $\omega$ Top $\hookrightarrow \omega$ Equ.

Lemma 3. If $Z$ is tight-injective and $f: X \rightarrow Y$ is tight then $Z^{f}: Z^{Y} \rightarrow Z^{X}$ is tight.

Proof. By Lemma 2.1.

Lemma 4. Given tight maps $e: X \rightarrow Z$ and $f: Y \rightarrow W$, where $Y$ is in $\omega \mathbf{0 E q u}$ and $Z, W$ are in $\omega \mathbf{E q u}_{\mathbf{t i}}$, then the function $f^{e^{-1}}:|Y|^{|X|} \rightarrow|W|^{|Z|}$ (using the bijectivity of e) restricts to a function $g:\left|Y^{X}\right| \rightarrow\left|W^{Z}\right|$, and this gives a tight morphism $g: Y^{X} \rightarrow W^{Z}$.

Proof. Given $e$ and $f$ as in the statement, consider the pullback in $\omega \mathbf{E q u}$ below.

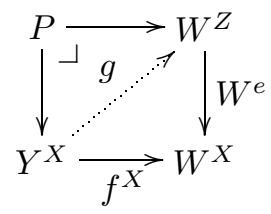

Then $W^{e}$ is tight by Lemma $3, f^{X}$ is tight by Lemma 1.5 , and so the other two maps are tight by Lemma 1.4. By Lemma $2.1, W^{Z}$ is tight-injective, so there exists a unique morphism $g: Y^{X} \rightarrow W^{Z}$ making the top-left triangle commute, whose function is easily checked to be the restriction of $f^{e^{-1}}:|Y|^{|X|} \rightarrow|W|^{|Z|}$. It remains to show that $g$ is tight. For this, note that the bottom-right triangle commutes because the left edge is tight, hence epi. Thus $g$ is mono because it right-factors the mono $f^{X}$. Finally, $g$ projects every space in $\omega \mathbf{0 E q u}$ because the tight map $P \longrightarrow W^{Z}$ does, and $g$ left-factors this.

Lemma 5. If $\operatorname{order}(\sigma) \leq 1$ then $\left([\sigma)_{I}\right.$ is isomorphic to an equilogical space $X$ with $\|X\|=\mathbb{N}^{\omega}$, hence $\left([\sigma]_{I}\right.$ is in $\omega \mathbf{0 E q u}$.

Proof. We illustrate when $\sigma$ is real $\rightarrow$ real, which is typical. The underlying space of $(\text { real })_{I}=\mathrm{R}_{\mathrm{I}}$ is $\mathbb{N} \times 3^{\omega}$. By Proposition 6, the exponential $\mathrm{R}_{\mathrm{I}} \mathrm{R}_{\mathrm{I}}$ is isomorphic to an object with underlying space a subspace $R \subseteq W$, where $W$ is a countablybased $\omega$-projecting cover of the the space $\left(\mathbb{N} \times 3^{\omega}\right)^{\left(\mathbb{N} \times 3^{\omega}\right)}$ in $\omega$ qTop. But, using Proposition 3 , we can calculate this exponential in $\omega \mathbf{q T o p}$ by:

$$
\begin{aligned}
&\left(\mathbb{N} \times 3^{\omega}\right)^{\left(\mathbb{N} \times 3^{\omega}\right)} \cong\left(\mathbb{N} \times 2^{\mathbb{N}}\right)^{\left(\mathbb{N} \times 2^{\mathbb{N}}\right)} \cong \mathbb{N}^{\left(\mathbb{N} \times 2^{\mathbb{N}}\right)} \times\left(2^{\mathbb{N}}\right)^{\left(\mathbb{N} \times 2^{\mathbb{N}}\right)} \\
& \cong\left(\mathbb{N}^{2^{\mathbb{N}}}\right)^{\mathbb{N}} \times 2^{\mathbb{N} \times \mathbb{N} \times 2^{\mathbb{N}}} \cong \mathbb{N}^{\mathbb{N}} \times 2^{\mathbb{N} \times 2^{\mathbb{N}}} \\
& \cong \mathbb{N}^{\mathbb{N}} \times\left(2^{2^{\mathbb{N}}}\right)^{\mathbb{N}} \cong \mathbb{N}^{\mathbb{N}} \times \mathbb{N}^{\mathbb{N}} \cong \mathbb{N}^{\mathbb{N}} \cong \mathbb{N}^{\omega}
\end{aligned}
$$

This is Baire space, which is already countably-based, thus one can take $W=\mathbb{N}^{\omega}$ itelf, meaning that $q$ in diagram (2) is an isomorphism. Consequently, so is its pullback $q^{\prime}$. Also, $\mathrm{R}_{\mathrm{I}}$ satisfies the conditions for $Y$ in Proposition 7, thus $F \subseteq\left(\mathbb{N} \times 3^{\omega}\right)^{\left(\mathbb{N} \times 3^{\omega}\right)}$ is closed, hence $R \subseteq \mathbb{N}^{\omega}$ is closed. As $R$ is nonempty and 
$\mathbb{N}^{\omega}$ is countably based, $R$ is a retract of $\mathbb{N}^{\omega}$ by Proposition 2. So ([real $\rightarrow$ real $)$ is isomorphic to an object with underlying space a retract of $\mathbb{N}^{\omega}$, hence it is isomorphic to an object with underlying space $\mathbb{N}^{\omega}$ itself.

Theorem 2 is an immediate consequence of:

Lemma 6. If $\operatorname{order}(\sigma) \leq 2$ then $(\sigma)_{I}=(\sigma)_{E}$ and the identity function gives a tight morphism $\left([\sigma)_{I} \rightarrow\left([\sigma]_{E}\right.\right.$.

Proof. By induction on $\sigma$. The interesting case is $\sigma_{1} \rightarrow \sigma_{2}$. By the induction hypothesis, $\left(\sigma_{1}\right)_{I}=\left(\sigma_{1}\right)_{E},\left(\sigma_{2}\right)_{I}=\left(\sigma_{2}\right)_{E}$ and identity functions gives tight morphisms $\left(\left[\sigma_{1}\right)_{I} \rightarrow\left(\left[\sigma_{1}\right)_{E}\right.\right.$ and $\left(\left[\sigma_{2}\right)_{I} \rightarrow\left(\left[\sigma_{2}\right)_{E}\right.\right.$. Moreover, $\left(\left[\sigma_{1}\right)_{E}\right.$ and $\left(\left[\sigma_{2}\right)_{E}\right.$ are both tight-injective, as remarked below Lemma 2 . Because order $(\sigma) \leq 2$, it holds that $\operatorname{order}\left(\sigma_{1}\right) \leq 1$, hence $\left(\left[\sigma_{1}\right)_{I}\right.$ is in $\omega \mathbf{0 E q u}$, by Lemma 5 . The conditions are thus fulfilled for applying Lemma 4 , which gives the identity function as a tight morphism $\left([\sigma)_{I} \rightarrow\left([\sigma)_{E}\right.\right.$, hence $(\sigma)_{E}=(\sigma)_{I}$.

We end this section by considering how Theorem 2 might be extended to higher types. Certainly, the proof above does not extend directly, because one can show that $\left([(\text { real } \rightarrow \text { real }) \rightarrow \text { real })_{I}\right.$ is not in $\omega \mathbf{0 E q u}$. However, this leaves open the possibility of replacing the use of $\omega \mathbf{0 E q u}$ with that of another category.

Proposition 8. Suppose there exists a full subcategory of $\omega \mathbf{E q u}$ satisfying four conditions: (i) it is closed under finite products; (ii) it contains $\mathbb{N}^{+}$; (iii) it contains every object $(\sigma \sigma]_{I}$; (iv) every object is projective with respect to the "identity" $\mathrm{R}_{\mathrm{I}} \rightarrow \mathrm{R}_{\mathrm{E}}$. Then $(\sigma)_{E}=(\sigma)_{I}$ for all types $\sigma$.

We do not know whether such a subcategory exists. The difficult conditions to reconcile are (iii) and (iv). Let us pinpoint our ignorance more exactly by considering the "pure" second- and third-order types:

$$
\text { real }_{2} \equiv(\text { real } \rightarrow \text { real }) \rightarrow \text { real } \quad \text { real }_{3} \equiv \text { real }_{2} \rightarrow \text { real }
$$

\section{Proposition 9.}

1. $\left(\text { real }_{3}\right)_{E} \supseteq\left(\text { real }_{3}\right)_{I}$.

2. $\left(\mathrm{real}_{3}\right)_{E}=\left(\mathrm{real}_{3}\right)_{I}$ if and only if the object $\left(\left[\mathrm{real}_{2}\right]_{I}\right.$ is projective with respect to the identity $\mathrm{R}_{\mathrm{I}} \rightarrow \mathrm{R}_{\mathrm{E}}$ (tracked by $\mathrm{r}$ ).

Proof. 1. We refer to the diagram below

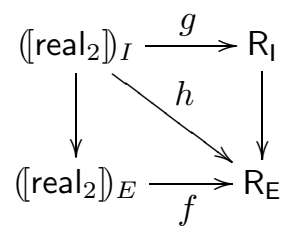

where the left-hand map is the tight morphism given by Lemma 6 . Because $\mathrm{R}_{\mathrm{E}}$ is tight-injective, for every $g$ there exists a unique $f$ making the outer square commute. Thus $\left(\text { real }_{3}\right)_{E} \supseteq\left(\text { real }_{3}\right)_{I}$. 
2. For, the 'if' direction, assume that $\left(\left[\text { real }_{2}\right)_{I}\right.$ is projective with respect to $\mathrm{R}_{\mathrm{I}} \rightarrow \mathrm{R}_{\mathrm{E}}$. Then, for every $f$, there exists a unique $g$ making the outer square commute. Thus indeed $\left(\mathrm{real}_{3}\right)_{E}=\left(\mathrm{real}_{3}\right)_{I}$.

Conversely, suppose that $\left(\text { real }_{3}\right)_{E}=\left(\text { real }_{3}\right)_{I}$, and consider any morphism $h$ as in the diagram. Because the left-hand edge is tight-injective, there exists a unique $f$ making the bottom-left triangle commute. By the assumed equality, it follows that there exists a unique $g$ making the outside square commute, and hence the top-right triangle commutes. The existence of $g$ thus establishes projectivity.

We have not succeeded in establishing whether $\left(\left[\text { real }_{2}\right]_{I}\right.$ is projective with respect to the identity $R_{I} \rightarrow R_{E}$. However, we have managed to reduce this condition to a conjecture concerning the topology of the Kleene-Kreisel continuous functionals over $\mathbb{N}$ [Kle59,Kre59]. Many presentations of the continuous functionals are known, but, for our conjecture, the simplest description is as the hierarchy of simple types over $\mathbb{N}$ in the cartesian-closed category $\omega \mathbf{q T o p}$, or equivalently in Seq, or equivalently in the cartesian-closed category of compactly-generated Hausdorff spaces, see [Nor80]. Our conjecture concerns the continuous functionals of pure second-order type.

Conjecture 1. The sequential space $\mathbb{N}^{\mathbb{B}}$, where $\mathbb{B}=\mathbb{N}^{\mathbb{N}}$, is zero dimensional.

Actually, we do not even know whether $\mathbb{N}^{\mathbb{N}^{\mathbb{N}}}$ is regular. The only positive facts we know about this space, in addition to what we have already stated, is that it is a totally-disconnected Hausdorff space in which every closed set is $G_{\delta}$ (i.e. is a countable intersection of open sets). On the negative side, $\mathbb{N}^{\mathbb{N}^{\mathbb{N}}}$ is not countably based, indeed it is not even first-countable.

Proposition 10. If Conjecture 1 holds then $\left(\text { real }_{2}\right]_{I}$ is projective with respect to the identity $\mathrm{R}_{\mathrm{I}} \rightarrow \mathrm{R}_{\mathrm{E}}$ and hence $\left(\mathrm{real}_{3}\right)_{E}=\left(\mathrm{real}_{3}\right)_{I}$.

Proof. By Lemma 5, $\left([\text { real })_{I}\right.$ and $\left([\text { real } \rightarrow \text { real })_{I}\right.$ are isomorphic to an object of the form $\left(\mathbb{R}, \mathbb{N}^{\omega}, r_{0}\right)$ and $\left((\text { real } \rightarrow \text { real })_{I}, \mathbb{N}^{\omega}, r_{1}\right)$ respectively. By Proposition 6 , $\left(\left(\text { real }_{2}\right)_{I}\right.$ is thus isomorphic to an object $\left(\left(\text { real }_{2}\right)_{I}, R, q^{\prime \prime} \circ q^{\prime}\right)$ where $q^{\prime}$ is obtained by pullback:

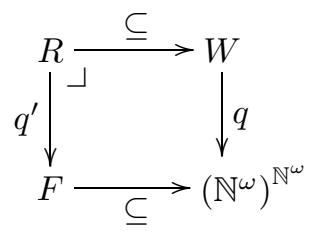

where $q$ an $\omega$-projecting cover of $\left(\mathbb{N}^{\omega}\right)^{\mathbb{N}^{\omega}}$ by a countably based $W$, so $q^{\prime}$ is $\omega$ projecting and hence a topological quotient.

By Proposition 3, we have that $\left(\mathbb{N}^{\omega}\right)^{\mathbb{N}^{\omega}} \cong\left(\mathbb{N}^{\mathbb{N}}\right)^{\mathbb{N}^{\mathbb{N}}} \cong \mathbb{N}^{\left(\mathbb{N} \times \mathbb{N}^{\mathbb{N}}\right)} \cong \mathbb{N}^{\mathbb{N}}$. Thus, by Conjecture $1,\left(\mathbb{N}^{\omega}\right)^{\mathbb{N}^{\omega}}$ is zero-dimensional. Moreover, by Proposition $6, F$ is a closed subspace of $\left(\mathbb{N}^{\omega}\right)^{\mathbb{N}^{\omega}}$. Thus $F$ is also zero-dimensional. 
To prove that $\left(\left(\text { real }_{2}\right)_{I}\right.$ is projective with respect to the $\mathrm{R}_{\mathrm{I}} \rightarrow \mathrm{R}_{\mathrm{E}}$, consider any morphism $f:\left(\left[\text { real }_{2}\right]\right)_{I} \rightarrow \mathrm{R}_{\mathrm{E}}$. We must find a morphism $\bar{f}:\left(\left[\text { real }_{2}\right]_{I} \rightarrow \mathrm{R}_{\mathrm{I}}\right.$ making the digram below commute.

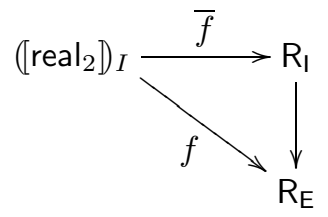

Using the isomorphic representation of $\left(\left[\text { real }_{2}\right)_{I}\right.$ above, the function $f$ is tracked by a continuous $g: R \rightarrow \mathbb{R}$. As $\mathrm{R}_{\mathrm{E}}$ is in the inclusion of $\omega \mathbf{T o p}$ in $\omega \mathbf{E q u}$, we have that $g=f \circ q^{\prime \prime} \circ q$.

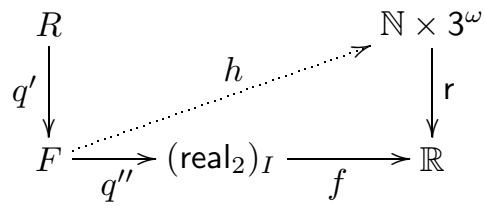

Now $f \circ q^{\prime \prime} \circ q$ is continuous, and $q^{\prime}$ is a quotient map, so $f \circ q^{\prime \prime}$ is continuous. By Proposition 4 , there exists a continuous $h$ making the triangle above commute. But then $h \circ q^{\prime}$ tracks the required morphism $\bar{f}$.

There are also connections between the possible zero-dimensionality of the spaces of continuous functionals at all types and the agreement of $([\sigma])_{E}$ and $(\sigma \sigma)_{I}$ for arbitrary $\sigma$. However, these are less straightforward to state.

\section{Proof of Theorem 1}

We prove Theorem 1 by reducing it to Theorem 2. This requires some work. Although we have stated that $\omega \mathbf{E q u}$ is equivalent to the full subcategory $\operatorname{Asm}(\omega \mathbf{L})$ of $\operatorname{Asm}(\omega \mathbf{B C})$, this is of no immediate help because neither $\llbracket$ real $\rrbracket_{E}$ nor $\llbracket$ real $\rrbracket_{I}$ resides in this subcategory. However, following [Bau00], there is a second way of viewing $\mathbf{A s m}(\omega \mathbf{L})$, and hence $\omega \mathbf{E q u}$, as (equivalent to) a full subcategory of $\operatorname{Asm}(\omega \mathbf{B C})$, under which $\llbracket$ real $\rrbracket_{E}$ and $\llbracket$ real $\rrbracket_{I}$ are included.

There are three interesting functors between $\mathbf{A s m}(\omega \mathbf{L})$ and $\mathbf{A s m}(\omega \mathbf{B C})$, as depicted below.

$$
\begin{gathered}
\operatorname{Asm}(\omega \mathbf{B C}) \\
I \uparrow \dashv T \downarrow \dashv K \\
\mathbf{A s m}(\omega \mathbf{L})
\end{gathered}
$$

Here $I$ is the full and faithful subcategory inclusion. Its faithful left adjoint $T$ is defined by simply adding a top element to $\|A\|$ to obtain the lattice $\|T(A)\|$ 
and leaving the other components unchanged. Finally, the full and faithful $K$, which is left-adjoint to $T$, maps an assembly $A$ in $\mathbf{A s m}(\omega \mathbf{L})$ to the assembly $K(A)$ obtained by defining $\|K(A)\|=\operatorname{cl}(\operatorname{supp}(A))$, where

$$
\begin{aligned}
& \operatorname{supp}(A) \quad=\{x \in\|A\| \mid \text { there exists } a \in|A| \text { such that } x \Vdash a\}, \\
& \operatorname{cl}(E)=\{z \in\|A\| \mid \text { for all } y \ll z \text { there exists } x \in E \text { with } y \sqsubseteq z\},
\end{aligned}
$$

again with the other components left unchanged. Note that $\operatorname{cl}(E)$ is the closure of $E$ in the Scott topology on $\|A\|$. We say that an assembly $A$ in $\operatorname{Asm}(\omega \mathbf{B C})$ is dense if $\operatorname{supp}(A)$ is dense in $\|A\|$, and essentially dense if it is isomorphic to a dense assembly. The image of $K$ is always a dense assembly, moreover $K$ and $T$ cut down to give an equivalence between $\operatorname{Asm}(\omega \mathbf{L})$ and the full subcategory, $\mathbf{A s m}_{\mathbf{e d}}(\omega \mathbf{B C})$, of $\mathbf{A s m}(\omega \mathbf{B C})$ on essentially dense assemblies [BBS02,Bau00].

Proposition 11. For any type $\sigma$,

1. $\llbracket \sigma \rrbracket_{E}$ is a dense assembly, and

2. if $\operatorname{order}(\sigma) \leq 1$ then $\llbracket \sigma \rrbracket_{I}$ is an essentially dense assembly.

Proof. 1. This follows from Normann's density theorem for an $\omega$-algebraic variant of the interval domain [Nor00b], along with the fact that $\mathcal{I}$ is a retract of this algebraic domain.

2. This is delayed to Sect. 8 .

Lemma 7. For any type $\sigma$,

1. $[\sigma]_{E}=(\sigma)_{E}$, and

2. if $\operatorname{order}(\sigma) \leq 2$ then $[\sigma]_{I}=(\sigma)_{I}$.

Proof. As mentioned above, the functor $T: \mathbf{A s m}_{\mathbf{e d}}(\omega \mathbf{B C}) \rightarrow \mathbf{A s m}(\omega \mathbf{L})$ is an equivalence of categories, and hence preserves cartesian-closed structure. In general, the cartesian-closed structure of $\mathbf{A s m}_{\mathbf{e d}}(\omega \mathbf{B C})$ is not inherited from the larger category $\mathbf{A s m}(\omega \mathbf{B C})$. However, given two essentially dense assemblies $A, B$, if $B^{A}$ in $\mathbf{A} \mathbf{s m}(\omega \mathbf{B C})$ is essentially dense, then $B^{A}$ is necessarily also the exponential in $\mathbf{A s m}_{\mathbf{e d}}(\omega \mathbf{B C})$. Thus, statement 1 follows from Proposition 11.1, as long as the composite of equivalences $\mathbf{A s m}_{\mathbf{e d}}(\omega \mathbf{B C}) \stackrel{T}{\longrightarrow} \operatorname{Asm}(\omega \mathbf{L}) \stackrel{F}{\longrightarrow} \omega \mathbf{E q u}$ maps $\llbracket$ real $\rrbracket_{E}$ to an object isomorphic to $\mathrm{R}_{\mathrm{E}}$. It is easily verified that this is indeed the case.

For statement 2, by Proposition 11.2, a similar remark applies to $\sigma$ with $\operatorname{order}(\sigma) \leq 1$, as long as the composite $F T$ maps $\llbracket$ real $\rrbracket_{I}$ to an equilogical space isomorphic to $\mathrm{R}_{\mathrm{I}}$. We verify that this is the case. Using the definition of $F$ from Sect. 4, one calculates that $\| F T \llbracket$ real $\rrbracket_{I} \|$ is homeomorphic to the subspace of real-representing elements in $\mathcal{J}$ under the Scott topology. It is routine to verify that this is, in turn, homeomorphic to $\mathbb{N} \times 3^{\omega}$, and that the induced surjection $q_{\left(F T \llbracket \text { real } \rrbracket_{I}\right)}: \| F T \llbracket$ real $\rrbracket_{I} \| \rightarrow \mathbb{R}$ is isomorphic over $\mathbb{R}$ to $\mathrm{r}: \mathbb{N} \times 3^{\omega} \rightarrow \mathbb{R}$.

It remains to establish statement 2 for $\sigma$ with $\operatorname{order}(\sigma)=2$. This can be reduced to the only interesting case, in which $\sigma$ is of the form $\sigma_{1} \rightarrow \sigma_{2}$, with both 
$\sigma_{1}$ and $\sigma_{2}$ of order 1 . Then, by the proof above for such types, $F T \llbracket \sigma_{1} \rrbracket_{I} \cong\left(\left[\sigma_{1}\right]_{I}\right.$ and $F T \llbracket \sigma_{2} \rrbracket_{I} \cong\left(\left[\sigma_{2}\right)_{I}\right.$. But then $\left[\sigma_{1} \rightarrow \sigma_{2}\right]_{I}$ is the hom-set from $\llbracket \sigma_{1} \rrbracket_{I}$ to $\llbracket \sigma_{2} \rrbracket_{I}$ in $\mathbf{A s m}(\omega \mathbf{B C})$, hence in $\mathbf{A s m}_{\mathbf{e d}}(\omega \mathbf{B C})$. Similarly, $\left(\sigma_{1} \rightarrow \sigma_{2}\right)_{I}$ is the hom-set from $\left(\left[\sigma_{1}\right)_{I}\right.$ to $\left(\left[\sigma_{2}\right]_{I}\right.$ in $\omega \mathbf{E q u}$, which, by the above isomorphisms, is identical to the hom-set from $F T \llbracket \sigma_{1} \rrbracket_{I}$ to $F T \llbracket \sigma_{2} \rrbracket_{I}$. But, as $F T: \mathbf{A s m}_{\mathbf{e d}}(\omega \mathbf{B C}) \rightarrow \omega \mathbf{E q u}$ is an equivalence of categories, it is full and faithful, so the two hom-sets coincide. Thus indeed $\left(\sigma_{1} \rightarrow \sigma_{2}\right)_{I}=\left[\sigma_{1} \rightarrow \sigma_{2}\right]_{I}$.

Theorem 1 follows immediately from Lemma 7 and Theorem 2 .

The above proof of Lemma 7.2 generalizes to show that if Proposition 11.2 holds for types $\sigma$ with $\operatorname{order}(\sigma) \leq n$ then Lemma 7.2 holds for types $\sigma$ with $\operatorname{order}(\sigma) \leq n+1$. However, for second-order types, we do not even know whether the property of Lemma 7.2 holds for the pure type real 2 of Sect. 6 .

\section{Extensionalization}

In this section, we prove Proposition 11.2. Our proof establishes a property of the domains underlying $\llbracket \sigma \rrbracket_{I}$, for first-order $\sigma$, that we call extensionalization. This property is of interest independent of its application to Proposition 11.2.

Following [Ber93], we define the set of total elements $\mathcal{T}_{\tau} \subseteq\|\tau\|$, where $\|\tau\|$ is the domain interpreting a PCF type $\tau$. This is by:

$$
\begin{aligned}
\mathcal{T}_{\text {nat }} & =\mathbb{N} \subseteq \mathbb{N}_{\perp} \\
\mathcal{T}_{\tau_{1} \times \tau_{2}} & =\mathcal{T}_{\tau_{1}} \times \mathcal{T}_{\tau_{2}} \\
\mathcal{T}_{\tau_{1} \rightarrow \tau_{2}} & =\left\{f \in\left\|\tau_{1} \rightarrow \tau_{2}\right\| \mid \text { for all } x \in \mathcal{T}_{\tau_{1}}, f(x) \in \mathcal{T}_{\tau_{2}}\right\}
\end{aligned}
$$

Recall also that, for a type $\sigma$ over real, $\left\|\llbracket \sigma \rrbracket_{I}\right\|=\left\|\sigma^{*}\right\|$, where $(\cdot)^{*}$ is the translation to PCF types from Sect. 3. The proof of the next proposition uses Berger's generalization of the "KLS Theorem" [Ber93].

Proposition 12 (Extensionalization). For any $\sigma$ with $\operatorname{order}(\sigma) \leq 1$, the identity function on $\llbracket \sigma \rrbracket_{I}$ is tracked by a function $i:\left\|\llbracket \sigma \rrbracket_{I}\right\| \rightarrow\left\|\llbracket \sigma \rrbracket_{I}\right\|$ with the property that, for all $x \in \mathcal{T}_{\sigma^{*}}, \quad i(x) \in \mathcal{T}_{\sigma^{*}} \cap \operatorname{supp}\left(\llbracket \sigma \rrbracket_{I}\right)$.

Proof. Easily, $\operatorname{cl}\left(\operatorname{supp}\left(\llbracket\right.\right.$ real $\left.\left.\rrbracket_{I}\right)\right)=\{f \in \mathcal{J} \mid$ for all $n \geq 1, f(n) \in\{\perp, 0,1,2\}\}$, and we write $\mathcal{J}^{\prime}$ for this subdomain of $\mathcal{J}$. It is convenient to reinterpret the intensional hierarchy, using $\mathcal{J}^{\prime}$ in place of $\mathcal{J}$. To this end, define $\llbracket$ real $\rrbracket_{I}^{\prime}$ to be the assembly with underlying domain $\mathcal{J}^{\prime}$, and with the other components as for $\llbracket$ real $\rrbracket_{I}$, and then define $\llbracket \sigma \rrbracket_{I}^{\prime}$ using the cartesian-closed structure of $\mathbf{A s m}(\omega \mathbf{B C})$. Also, define Write $\|\sigma\|^{\prime}$ for the underlying domains $\left\|\llbracket \sigma \rrbracket^{\prime}\right\|$. We again define sets of total elements $\mathcal{T}_{\sigma}^{\prime} \subseteq\|\sigma\|^{\prime}$ by: $\mathcal{T}_{\text {real }}^{\prime}=\{f \mid$ for all $n, f(n) \neq \perp\}$, with $\mathcal{T}_{\sigma_{1} \times \sigma_{2}}^{\prime}$ and $\mathcal{T}_{\sigma_{1} \rightarrow \sigma_{2}}^{\prime}$ determined as above. We show that the following facts hold.

1. For all $\sigma$, there is an embedding-retraction pair given by $e:\|\sigma\|^{\prime} \rightarrow\left\|\sigma^{*}\right\|$ and $r:\left\|\sigma^{*}\right\| \rightarrow\|\sigma\|^{\prime}$ such that, for all $x \in \mathcal{T}_{\sigma}^{\prime}, e(x) \in \mathcal{T}_{\sigma^{*}}$, and, for all $x \in$ $\mathcal{T}_{\sigma^{*}}, r(x) \in \mathcal{T}_{\sigma}^{\prime}$. Moreover, these retractions track "identity" isomorphisms between $\llbracket \sigma \rrbracket_{I}^{\prime}$ and $\llbracket \sigma \rrbracket_{I}$. 
2. For any $\sigma$ with $\operatorname{order}(\sigma) \leq 1$, the identity function on $\llbracket \sigma \rrbracket_{I}^{\prime}$ is tracked by a function $i:\|\sigma\|^{\prime} \rightarrow\|\sigma\|^{\prime}$ with the properties that, for all $x \in \mathcal{T}_{\sigma}^{\prime}, \quad i(x) \in$ $\mathcal{T}_{\sigma} \cap \operatorname{supp}\left(\llbracket \sigma \rrbracket_{I}^{\prime}\right)$.

The proposition follows easily by using the retractions to transport property 2 above to the original $\llbracket \sigma \rrbracket_{I}$ case.

Property 1 is easily established by induction on $\sigma$, so we just prove 2 . For $\sigma$ with $\operatorname{order}(\sigma)=0$ this is trivial because $\operatorname{supp}\left(\llbracket \sigma \rrbracket_{I}\right)=\mathcal{T}_{\sigma}$ so $i$ can be the identity function. For first-order types, we illustrate with the typical case for $\sigma$ of real ${ }_{1} \equiv$ real $\rightarrow$ real. Easily $\operatorname{supp}\left(\llbracket\right.$ real $\left._{1} \rrbracket_{I}^{\prime}\right) \subseteq \mathcal{T}_{\text {real }_{1}}^{\prime}$, because $\operatorname{supp}\left(\llbracket \sigma \rrbracket_{I}\right)=\mathcal{T}_{\sigma}$.

Consider the diagram below.

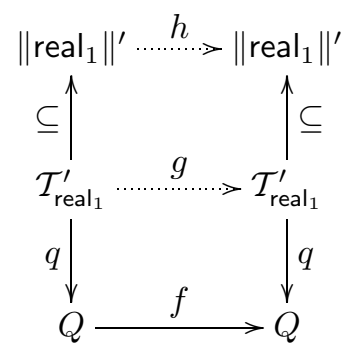

Here, $q$ is the topological quotient of $\mathcal{T}_{\text {real }_{1}}^{\prime}$, under the relative Scott topology from $\|$ real $_{1} \|$, by the consistency relation: $x \uparrow y$ iff there exists $z$ with $x \sqsubseteq z \sqsupseteq y$. It is known, [Ber93], that the following generalization of the "KLS Theorem" holds: for any continuous $f$ as in the diagram, there exist $g$ and $h$ making the squares commute. (This is a non-effective version of the "KLS Theorem" in [Ber93]. The proof uses the density of the inclusion $\mathcal{T}_{\text {real }}^{\prime} \subseteq \|$ real ${ }_{1} \|^{\prime}$.) However, $\mathcal{T}_{\text {real }}$ is homeomorphic to $\mathbb{N} \times 3^{\omega}$. This implies that $Q$ is homeomorphic to the exponential $\mathbb{N} \times 3^{\omega\left(\mathbb{N} \times 3^{\omega}\right)}$ in $\omega \mathbf{q T o p}$, which, as in the proof of Lemma 5 , is homeomorphic to $\mathbb{N}^{\omega}$.

One now calculates that the subspace $F \subseteq Q \cong \mathbb{N}^{\omega}$ consisting of those functions $p$ in the image of $q: \operatorname{supp}\left(\llbracket\right.$ real $\left._{1} \rrbracket_{I}^{\prime}\right) \subseteq \mathcal{T}_{\text {real }_{1}}^{\prime} \rightarrow Q$ is closed, and hence, by Proposition 2, a retract of $\mathbb{N}^{\omega}$ by a continuous function $r: \mathbb{N}^{\omega} \rightarrow F$. Then let $f$ be the composite $Q \cong \mathbb{N}^{\omega} \stackrel{r}{\rightarrow} F \subseteq Q$. As in the diagram, this gives $g$ and $h$, with $h$ being the $i$ required by statement 2 .

We call this result extensionalization for the following reason. As in Sect. 3, in order for an element $f \in \| \llbracket$ real $\rightarrow$ real $\rrbracket_{I} \|$ to track a morphism from $\llbracket$ real $\rrbracket_{I}$ to $\llbracket$ real $\rrbracket_{I}$ it must both preserve real-representing elements (i.e. it must preserve $\operatorname{supp}\left(\llbracket\right.$ real $\left.\left.\rrbracket_{I}\right)\right)$ and it must also preserve the equivalence between such representations; thus one might say that it must behave "extensionally". The proposition relates such "extensional" elements of $\| \llbracket$ real $\rightarrow$ real $\rrbracket_{I} \|$ to total ones. Firstly, because $i$ tracks the identity, it maps every extensional element $f$ to an equivalent total extensional one. Secondly, every non-extensional but total $f$ is mapped to an arbitrary extensional and still total element. Thus the total elements of 
$\| \llbracket$ real $\rightarrow$ real $\rrbracket_{I} \|$ are all "extensionalized" by $i$. Again we do not know whether such a process of extensionalization is also available for second-order $\sigma$ and above.

Corollary 1. If $\operatorname{order}(\sigma) \leq 1$ then the identity function on $\llbracket \sigma \rrbracket_{I}$ is tracked by a function $i$ such that, for all $x \in\left\|\llbracket \sigma \rrbracket_{I}\right\|, \quad i(x) \in \operatorname{cl}\left(\operatorname{supp}\left(\llbracket \sigma \rrbracket_{I}\right)\right)$.

Proof. By [Ber93], the inclusion $\mathcal{T}_{\sigma^{*}} \subseteq\left\|\llbracket \sigma \rrbracket_{I}\right\|$ is dense. Thus, taking $i$ as in Proposition 12,i $\left(\mathcal{T}_{\sigma^{*}}\right)$ is dense in the image of $i$. But, by Proposition $12, i\left(\mathcal{T}_{\sigma^{*}}\right) \subseteq$ $\operatorname{supp}\left(\llbracket \sigma \rrbracket_{I}\right)$. Thus indeed the image of $i$ is contained in $\operatorname{cl}\left(\operatorname{supp}\left(\llbracket \sigma \rrbracket_{I}\right)\right)$.

Proposition 11.2 follows, as the property stated in the corollary is easily seen to be sufficient to establish that $\llbracket \sigma \rrbracket_{I}$ is essentially dense.

\section{Eliminating Parallelism}

To conclude the paper, we return to our original motivation for studying the $\llbracket \sigma \rrbracket_{E}$ and $\llbracket \sigma \rrbracket_{I}$ hierarchies, namely that they correspond to the total functionals on reals definable in the two approaches to exact real-number computation. As discussed in Sect. 3, the $\llbracket \sigma \rrbracket_{E}$ functionals are exactly those programmable in $\Omega$ RealPCF + , and the functionals in $\llbracket \sigma \rrbracket_{I}$ are those programmable in $\Omega \mathbf{P C F}++$. Both these languages contain parallel primitives.

In the context of PCF, Normann has proved that the type hierarchies of total functionals over $\mathbb{N}$ programmable in $\mathbf{P C F}$ and $\mathbf{P C F}++$ are identical for arbitrary types [Nor00a]. By the same proof, the hierarchies of $\mathbb{N}$-functionals programmable in $\Omega \mathbf{P C F}$ and $\Omega \mathbf{P C F}++$ are identical. In other words, parallel primitives are unnecessary as far as programming total functionals over $\mathbb{N}$ is concerned. It is natural to ask whether a similar phenomenon of elimination of parallelism occurs also for total functionals over $\mathbb{R}$.

For the extensional approach, the situation is unsatisfactory. In [EHS99], it is proved that there is no sequential way of implementing even the first-order function of binary addition. For this reason, core RealPCF contains a primitive parallel-conditional operation. However, one may still question whether the parallel existential of RealPCF + is required for programming total functionals. Normann has investigated this question, and proved that all second-order functionals are definable in RealPCF relative to any extension, to partial reals, of the supremum-finding functional [Nor02]. He also proves that such extensions are, in general, strictly weaker than the parallel existential operator, although it remains an open question whether some such extension is already programmable in RealPCF.

Our final result is that, in the intensional approach, parallelism is eliminable up to type two. Recall, from Sect. 3, our notation for PCF and its semantics.

Theorem 3. If $\operatorname{order}(\sigma) \leq 2$ then, for any $f \in[\sigma]_{I}$, there exists an $\Omega \mathbf{P C F}$ program $P$ of type $\sigma^{*}$ such that $\llbracket P \rrbracket \Vdash_{\llbracket \sigma \rrbracket_{I}} f$.

Proof. Suppose order $(\sigma) \leq 2$. We first prove: 
if $x \Vdash_{\llbracket \sigma \rrbracket_{I}} f$ then there exists $y \in \mathcal{T}_{\sigma^{*}}$ such that $y \Vdash_{\llbracket \sigma \rrbracket_{I}} f$.

For zero- and first-order $\sigma$, this is immediate from Proposition 12. For secondorder $\sigma$, we consdier the typical case of $\sigma$ being real ${ }_{2}$ (as defined above Proposition 9). Let $i: \| \llbracket$ real $_{1} \rrbracket_{I}\|\rightarrow\| \llbracket$ real $_{1} \rrbracket_{I} \|$ be as given by Proposition 12 . Then, if $x \Vdash_{\llbracket \sigma \rrbracket_{I}} f$, it is immediate seen that $x \circ i \in \mathcal{T}_{\text {real }}{ }_{2}^{*}$, and $x \circ i \Vdash_{\llbracket \text { real }} \rrbracket_{I} f$.

To prove the theorem, suppose $x \Vdash_{\llbracket \sigma \rrbracket_{I}} f$. Then, by the above, there exists $y \in \mathcal{T}_{\sigma^{*}}$ such that $y \Vdash_{\llbracket \sigma \rrbracket_{I}} f$. As $y \in \mathcal{T}_{\sigma^{*}}$, by the non-computable version of Normann's theorem ("Berger's conjecture") [Nor00a], there exsists an $\Omega$ PCFterm $P$ of type $\sigma^{*}$ such that $\llbracket P \rrbracket$ is totally equivalent to $y$. But then one verifies that indeed $\llbracket P \rrbracket \Vdash_{\llbracket \sigma \rrbracket_{I}} f$.

Note thet the proof makes use of Normann's theorem for third-order PCFtypes - the first type level at which the theorem is interesting. Because of the type limitation on Proposition 12, we have no idea whether Theorem 3 holds for third-order types upwards.

To ease comparison with the results for the extensional case discussed above, we remark that we have also proved a version of Theorem 3 for the standard (oracle free) versions of $\mathbf{P C F}$ and $\mathbf{P C F}++$. Specifically, a total functional on $\mathbb{R}$ is definable in $\mathbf{P C F}$ if and only if it is definable in $\mathbf{P C F}++$. The proof involves writing PCF programs for the extensionalization functions $i$ of Proposition 12, and thus uses methods quite different from those of this paper.

\section{References}

[AJ94] S. Abramsky and A. Jung. Domain theory. In Handbook of Logic in Computer Science, volume 3, pages 1-168. OUP, 1994.

[Bau00] A. Bauer. The Realizability Approach to Computable Analysis and Topology. $\mathrm{PhD}$ thesis, Carnegie Mellon University, 2000.

[BBS02] A. Bauer, L. Birkedal, and D.S. Scott. Equilogical spaces. Theoretical Computer Science, to appear, 2002.

[Ber93] U. Berger. Total sets and objects in domain theory. Annals of Pure and Applied Logic, 60:91-117, 1993.

[DiG93] P. Di Gianantonio. A Functional Approach to Computability on Real Numbers. $\mathrm{PhD}$ thesis, Università di Pisa, 1993.

[Dug89] J. Dugundji. Topology. Wm. C. Brown, 1989.

[EE00] M.H. Escardó and A. Edalat. Integration in real PCF. Information and Computation, 160:128-166, 2000.

[EHS99] M.H. Escardó, M. Hofmann, and Th. Streicher. Mediation is inherently parallel. Talk at Workshop on Domains V, Darmstadt, 1999.

[ES99] M.H Escardó and Th. Streicher. Induction and recursion on the partial real line with applications to real PCF. Theoretical Computer Science, 210:121$157,1999$.

[Esc96] M.H. Escardó. PCF extended with real numbers. Theoretical Computer Science, 162:79-115, 1996.

[GL01] P. Gowland and D. Lester. A survey of exact computer arithmetic. In Computability and Complexity in Analysis. Springer LNCS 2064, 2001. 
[GS90] C.A. Gunter and D.S. Scott. Semantic domains. In Handbook of Theoretical Computer Science. Elsevier Science Publisher, 1990.

[Has] http://www.haskell.org/.

[Kle59] S.C. Kleene. Countable functionals. In Constructivity in Mathematics, pages 81-100. North-Holland, 1959.

[Kre59] G. Kreisel. Interpretation of analysis by means of functionals of finite type. In Constructivity in Mathematics, pages 101-128. North-Holland, 1959.

[Mac71] S. Mac Lane. Categories for the Working Mathematician. Springer-Verlag, 1971.

[MS02] M. Menni and A.K. Simpson. Topological and limit-space subcategories of countably-based equilogical spaces. Mathematical Structures in Computer Science, to appear, 2002.

[Nor80] D. Normann. Recursion on the countable functionals. Springer LNM 811, 1980.

[Nor00a] D. Normann. Computabilty over the partial continuous functionals. Journal of Symbolic Logic, 65:1133-1142, 2000.

[Nor00b] D. Normann. The continuous functionals of finite types over the reals. Electronic Notes in Theoretical Computer Science, 35, 2000.

[Nor02] D. Normann. Exact real number computations relative to hereditarily total functionals. Theoretical Computer Science, to appear, 2002.

[Plo77] G.D. Plotkin. LCF considered as a programming language. Theoretical Computer Science, 5(3):223-255, 1977.

[Sco96] D.S. Scott. A new category? Unpublished Manuscript. Available at http://www.cs.cmu.edu/Groups/LTC/, 1996.

[Sim98] A.K. Simpson. Lazy functional algorithms for exact real functionals. In Mathematical Foundations of Computer Science, pages 456-464. Springer LNCS 1450, 1998.

[WK87] K. Weihrauch and C. Kreitz. Representations of the real numbers and of the open subsets of the set of real numbers. Annals of Pure and Applied Logic, $35(3): 247-260,1987$. 